\title{
Accounting for diversity
}

\author{
Researchers, funders, database managers and journals all have a role to play in accounting for diversity and \\ prioritizing inclusion at the basic science level.
}

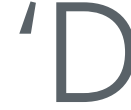
iversity' is a word that has been lately coming up a lot in the scientific community - and for good reason. Once largely dominated by white men, the global scientific community is certainly a lot more diverse today than even a few decades ago. But undoing a long history of bias will take more time and careful attention: hiring efforts should account for systemic racism, conference organizers should ensure that the speaker gender balance reflects the field of study, and research projects and funding focusing on particular regions of the world should be inclusive of local scientists, who bring a wealth of local knowledge, connections and lived experiences to the table.

Not only have white men historically dominated the scientific enterprise, medical science has also strongly favored research on the health of men and people of European ancestry. Much has been written about how the lack of focus on and research funding for diseases that affect mainly women or people of non-European ancestry has resulted in medical advances being unequally distributed. The failure of clinical trials evaluating drug treatments to be inclusive of different populations has resulted in harm and even death.

Diversity is also important at a more fundamental basic science level. It is a key variable inherent in the samples that researchers choose to analyze, and better attention needs to be paid to it. A lack diversity has been particularly apparent in human genetics studies: genome sequencing and genome-wide association studies have overwhelmingly used samples from individuals of European ancestry. Even when data from under-represented groups are available, researchers often discard it owing to concerns about statistical significance. While there is still a ways to go, good progress is being made to collect more genetic data from Asian and African populations via efforts such as GenomeAsia100K Project and the H3Africa consortium.

Inclusion of diverse populations is also vital in immunology studies. A recent study of SARS-CoV-2 showed that immune responses significantly differ by sex, with men exhibiting higher plasma levels of innate immune cytokines but women presenting more robust $\mathrm{T}$ cell responses. Researchers have also found that immune responses to bacterial pathogens are influenced by genetics, with individuals of African ancestry showing a stronger inflammatory response than those of European ancestry. Clearly, more work to understand how genetic differences underlie immune response is needed. The 10,000 Immunomes Project, which sought to robustly characterize the immunomes of a large, diverse, healthy human cohort, was a welcome step. And in this issue of Nature Methods we feature a Comment from a large group of researchers, representing countries around the world, who call for greater inclusion of diverse populations in immunogenomics studies of human adaptive immune responses.

The sex bias favoring research on men's health also extends to the preclinical and basic science levels: for example, male mice have dominated lab-based experiments. Concerns that the hormonal variability of female mice would complicate experimental procedures or confound results have led many researchers to avoid them altogether, to the detriment of studying the full picture of how various diseases or behaviors present. Encouragingly, large funders have recently taken notice. The National Institutes of Health in the United States has, since 2016, required that human and vertebrate animal projects it funds must take sex into account as a biological variable and that grant applications must make a strong justification when proposing to perform experiments using only one sex. The European Commission made a similar announcement last year, which will affect research that it funds via the Horizon Europe program.

Although such funder requirements are a welcome advance going forward, they do not address past failures to account for sex in research samples. At the cell line level, chromosomal sex has been largely ignored. A second Comment in this issue of Nature Methods is intended to draw readers' attention to a problem that has been largely hidden from view: not only do omics databases largely contain an over-representation of data from male human and animal subjects, but many popular omics resources do not annotate sample sex at all-which may influence the reliability of the tools built upon such resources and analyses utilizing such resources. To begin to resolve this troubling issue, the authors provide detailed recommendations for scientists, for databases and for funding agencies.

Journals have a role to play as well, in part by utilizing their platforms to highlight the scientific and societal need to account for diversity in research studies. Journals can also recommend or enforce research standards; for example, the Nature Portfolio journals recommend that authors follow the ARRIVE and SAGER guidelines for reporting sex in manuscripts. We are always happy to take further feedback from our readers about how we can better support diversity and inclusion in research for the benefit of all.

Published online: 7 June 2021

https://doi.org/10.1038/s41592-021-01193-5 